\title{
Enhanced Eco-Approach Control of Connected Electric Vehicles at Signalized Intersection with Queue Discharge Prediction
}

\author{
Haoxuan Dong, Weichao Zhuang, Boli Chen, Member, IEEE, Guodong Yin, Senior Member, IEEE,
} Yan Wang

\begin{abstract}
Long queues of vehicles are often found at signalized intersections, which increases the energy consumption of all the vehicles involved. This paper proposes an enhanced eco-approach control (EEAC) strategy with consideration of the queue ahead for connected electric vehicles ( $\mathrm{EV}$ s) at a signalized intersection. The discharge movement of the vehicle queue is predicted by an improved queue discharge prediction method (IQDP), which takes both vehicle and driver dynamics into account. Based on the prediction of the queue, the EEAC strategy is designed with a hierarchical framework: the upper-stage uses dynamic programming to find the general trend of the energy-efficient speed profile, which is followed by the lower-stage model predictive controller to computes the explicit solution for a short horizon with guaranteed safe inter-vehicular distance. Finally, numerical simulations are conducted to demonstrate the energy efficiency improvement of the EEAC strategy. Besides, the effects of the queue prediction accuracy on the performance of the EEAC strategy are also investigated.
\end{abstract}

Index Terms - electric vehicles, connected vehicles, eco-driving, energy efficiency, velocity planning, dynamic programming,

\section{INTRODUCTION}

$\mathrm{T}$ he growing transportation activities have been not only substantially enhancing the mobility of people and goods, but also producing more greenhouse gas (GHG) emissions and consuming a large amount of energy [1]. To reduce the vehicles' environmental impacts, tightening vehicle emission standards are introduced, which promotes the development of alternative vehicle propulsion [2] and energy storage systems [3]. Electric vehicles $(E V s)$ is one of the promising approaches to reduce the emissions of the transportation sector. However, its limited driving range and charging rate are still issues for large-scale promotion [4]. Another approach to achieve efficient vehicle operation is driving the vehicle at an energy-efficient speed, which is called the eco-driving in some literature [5]. Previous

Copyright (c) 2015 IEEE. Personal use of this material is permitted. However, permission to use this material for any other purposes must be obtained from the IEEE by sending a request to pubs-permissions@ieee.org.

This work was supported by Key R\&D Program of Jiangsu Province under Grants BE2019004, National Natural Science Funds for Distinguished Young Scholar under Grants 52025121, National Nature Science Foundation of China under Grants 51805081 and 51975118, UCL-ZJU Seed Funding 2020-21. (Corresponding author: Guodong Yin; Weichao Zhuang.)

H. Dong, W. Zhuang, G. Yin, Y. Wang are with the School of Mechanical Engineering, Southeast University, Nanjing 211189, China (e-mail: donghaox@foxmail.com; wezhuang@seu.edu.cn; ygd@seu.edu.cn; yanwangiv@outlook.com ).

B. Chen is with the Dept. of Electronic and Electrical Engineering at University College, London, UK (boli.chen@ucl.ac.uk). studies have indicated the energy-saving and emission reduction potentials of deploying the eco-driving system [6]. In general, researchers optimized the energy-efficient driving profile from two cases: highway cruising and urban driving.

On highways, the eco-driving system is designed to regulate the vehicle cruising speed for minimizing energy consumption. Some studies indicate that the rule-based strategy, e.g., smooth speed or pulse-and-glide operation has great potential in improving vehicle energy efficiency [7, 8]. However, the terrain and surrounding traffic information have a significant influence on vehicle operation, and neglecting them in controller design may lead to loss of potential of eco-driving. Thus, Zhuang et al. [9] designs a cruising strategy on the highway with varying slopes by using dynamic programming (DP) to improve electric vehicle energy efficiency and battery life simultaneously. Ding et al. [10] investigates the eco-cruising control while driving on winding roads. The optimal solution indicates that the vehicle should decelerate before entering a corner, sustain the optimal constant speed during turning, and accelerate out of the corner. In addition, Zhang et al. [11] proposes a model predictive control (MPC) based eco-driving strategy for hybrid electric vehicles to achieve efficient vehicle control by predicting the preceding vehicle velocity. Another study, presented by $\mathrm{Xu}$ et al. [12] proposes an instantaneous optimization method by converting vehicle kinetic energy into energy consumption to achieve cruising speed optimization.

The eco-driving strategy design in urban traffic complex due to the complicated conditions, for example, the traffic streams can be interrupted by the traffic signals. Recent developments in vehicle automation and connectivity technologies provide possibilities to obtain or predict the surrounding traffic conditions $[6,13]$. In particular, the signal phase and timing (SPaT) information of traffic signals can be obtained by vehicle-to-infrastructure (V2I) communication. Therefore, the eco-approach and departure strategy (EAD) at a signalized intersection has been designed based on different approaches. Katsaros et al. [14] develops a green light optimized speed advisory system (GLOSA) using deterministic and known SPaT information, to minimize average fuel consumption and stop delay at an intersection. Considering the uncertain of the traffic signal SPaT, Mahler et al. [15] introduces a signal-phase prediction model to determine the probability of green signals, then the best velocity trajectory is derived that maximizes the chance of going through an intersection. Lin et al. [16] studies 
energy-efficient control at multiple signalized intersections in a free-flow traffic environment, and presents a quasi-optimal rule to replace numerical solutions for real-time applications. However, EAD studies above generally assume that the vehicle is driving in a free-flow environment. The stopping events are determined not only by the traffic signal but also by preceding vehicles at the incoming intersection [17]. Thus, some studies investigated the EAD strategy considering preceding vehicles. Li et al. [18] considers the constraints arising from the preceding vehicles, a control method combining the safetyguaranteed and fuel-optimal operation is proposed. Xie et al. [19] uses the on-board radar to detect the movement of the preceding vehicle and developed an energy-saving control strategy. In addition, Ye et al. [20] designs an EAD strategy with consideration of predicted preceding vehicle, to ensure that the vehicle reaches the intersection stop line safely and energy efficiently. Bakibillah et al. [21] presents a learning-based method, which estimates the probability that the vehicle traveled through the intersection with moderated and nocongested traffic flow.

In reality, the movement of a vehicle may be interrupted or restricted by a vehicle queue rather than a single preceding vehicle while driving through a signalized intersection. By utilizing the information of the vehicle queue at the intersection through V2I technologies, He et al. [22] investigates the ecodriving strategy considering vehicle queue length. But the traffic information was given in a fully connected environment, the connectivity capabilities at all intersections and vehicles can only be realized in the distant future [23]. While, the vehicle queue ahead of the intersection changes over time, and the queue discharge movement is not easy to detect especially in partially connected environments [24]. Besides, most previous studies focused on the eco-driving control of conventional vehicles with an internal combustion engine. The energyefficient operation of EVs may be different because it could recover energy from regenerative braking.

Therefore, this paper will focus on the EV's EAD strategy design at the signalized intersection with consideration of vehicle queue, where the key is the queue discharge prediction. In the respect of vehicle speed optimization, there are a variety of queue discharge prediction methods available, including deterministic kinematic based method (DKM) $[25,26]$ and traffic flow based method [27, 28]. These methods mostly assume that all vehicles in the queue are simplified as a pointmass, and moving as a whole. Such assumptions ignore the dynamics of vehicles and drivers, i.e., acceleration and deceleration response time, startup delay and vehicle length, which are the main cause of queue oscillation [29]. In addition, Sun et al. [30] considers vehicle queue causes greater delay on the feasible traveling time window while the vehicle is passing through intersections, thus estimates queue delay via traffic data. However, a large amount of high-resolution traffic data is not easily accessible. Therefore, an accurate vehicle queue discharge prediction method is required to design an enhanced eco-approach control strategy (EEAC) in terms of energysaving and efficient traffic.

The major contributions of this paper are twofold. First, an improved queue discharge prediction method (IQDP) is proposed to predict the queue discharge movement for energyefficient speed planning, which considers the vehicle dynamics. Second, a hierarchical control framework is proposed. In the upper-stage, the DP algorithm is used to derive the long-term energy-efficient vehicle speed trajectory while considering the vehicle queue, followed by MPC deployed in the lower-stage, targeting the local adaptation of vehicle operation for safety purposes (account for the prediction error).

The remainder of this paper is organized as follows. Section II introduces vehicle dynamics and the problem of EAD at a signalized intersection considering vehicle queue. In Section III, the IQDP method is presented to predict the queue discharge movement. Section IV formulates the energy-efficient control problem and solves it by using DP and linear time-varying MPC (LTV-MPC). The performance of queue discharge prediction and energy efficiency is evaluated by simulation in Section V. Section VI concludes this paper.

\section{VEHICLE DYNAMICS AND PROBLEM FORMULATION}

\section{A. Vehicle Dynamics}

For the EAD study, only the vehicle longitudinal dynamics is modeled, as expressed in (1),

$$
\begin{aligned}
& \dot{x}(t)=f(u(t), x(t))= \\
& {\left[\begin{array}{ll}
0 \\
0 & \left.-\frac{g f \cos \theta}{v(t)}-\frac{g \sin \theta}{v(t)}-\frac{0.5 C_{D} A \rho v(t)}{m}\right] x(t)+\left[\begin{array}{l}
0 \\
\frac{1}{m}
\end{array}\right] u(t)
\end{array}\right.}
\end{aligned}
$$

where $x$ is the state variable, $x=\left[\begin{array}{ll}d & v\end{array}\right]^{T} . d$ and $v$ are the travel distance and vehicle speed, respectively. $u$ is the control variable, which is the vehicle traction force $F$, i.e., positive for propulsion and negative for braking. $g$ is the acceleration of gravity, $f$ is the rolling resistance coefficient, $m$ is the vehicle mass, $C_{D}$ is the aerodynamic drag coefficient, $A$ is the frontal area, $\rho$ is the air density, and $\theta$ is the road gradient.

This paper optimizes the EAD strategy of four-wheel-drive (4WD) EVs, which is powered by four identical in-wheel motors (IWM). The layout of 4WD EVs is shown in Fig. 1. The torques on each wheel can be controlled independently in 4WD EVs, which enables advantages in terms of vehicle dynamics control [31], energy optimization [32], and vehicle structural flexibility [33].

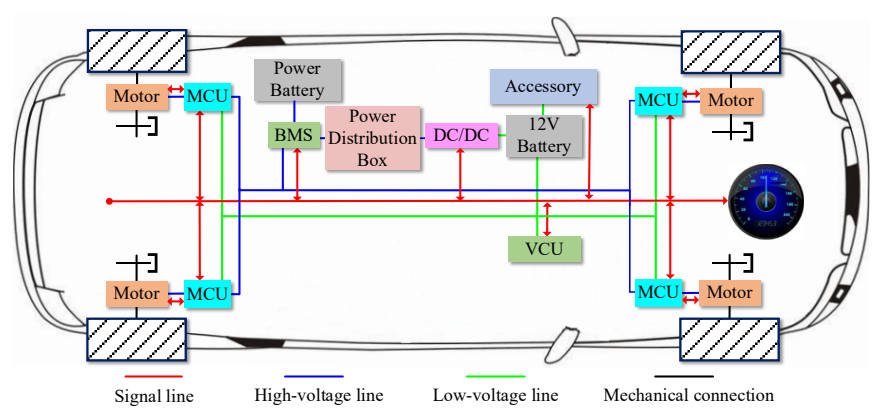

Fig. 1. The layout of $4 \mathrm{WD}$ EVs. Here MCU, VCU, and BMS represent the motor control unit, vehicle control unit, and battery management system, respectively. 
This paper focuses on eco-driving control in the context of daily driving. There is some evidence [34] that everyday drivers use accelerations remarkably smaller than adherence limits for driving comfort and safety purposes. Hence, we assume the wheels do not slip at their contact point with the ground but can rotate freely about their axes of rotation. Such assumption is usually invoked in eco-driving and energy management studies in the literature $[6,28]$. Under the non-slip assumption, the vehicle force during the traction process is only supplied by IWM. Otherwise, the EVs have the function of regenerative braking, then the vehicle barking force including motor and friction braking force. The relationship of the vehicle force, motor force, and friction braking force is shown in (2),

$$
F=\left\{\begin{array}{cc}
\sum F_{m} & \text { if } F \geq 0 \\
F_{b f}+\sum F_{m} & \text { if } F<0
\end{array}\right.
$$

where $F_{m}$ the motor force, positive for propulsion and negative for generating. $F_{b f}$ is the friction braking force.

The power of the IWM is calculated by (3),

$$
P_{m}=F_{m} \frac{2 \pi r_{w} n}{60}
$$

where $P_{m}$ is the motor power for each wheel, $n$ is the wheel rotational speed, and $r_{w}$ is the radius of wheels. Then, the required battery power $P_{b}$ is the sum of four IWMs and auxiliary power $P_{a}$, i.e.,

$$
P_{b}=P_{a} \eta_{b}^{-1}+\sum P_{m} \eta_{m}^{-\operatorname{sign}\left(P_{m}\right)} \eta_{b}^{-\operatorname{sign}\left(P_{m}\right)}
$$

where $\eta_{m}$ is the motor efficiency which is determined by the motor torque and speed as shown in Fig. 2. $\eta_{b}$ is the battery efficiency, and $\operatorname{sign}(\cdot)$ is the signum function.

In addition, the energy consumption of 4WD EVs is affected by the driving and braking torque allocation strategy. In this paper, the driving torque of each wheel is allocated based on the axle load, which is expressed in detail in [35], and the ideal regenerative braking strategy proposed in [36] is used in the braking control.

\section{B. Problem Formulation}

The EAD strategy at the signalized intersection is recognized as a promising technique to reduce vehicle energy consumption in urban traffic. The simplest EAD strategy (known as GLOSA in some literature [14]) targets the vehicle speed range that enables the vehicle to drive into the intersection on green signal.

As shown in Fig. 3(a), zone A is the derived vehicle speed range without stop-and-go maneuver by using the SPaT information of the traffic signal. By eliminating the stop-andgo maneuvers at a signalized intersection, significant vehicle energy consumption reduction can be achieved. In addition, many studies focused on the optimization of vehicle speed trajectory to achieve the energy-efficient driving operation, as the blue solid line in Fig. 3(a). The strategy which calculates the vehicle speed is referred to as the regular EAD in the following.

However, the signalized intersection typically introduces vehicle stops along a trip thus form the vehicle queue, as shown in Fig. 3(b), the energy-efficient speed trajectory maybe not feasible due to the vehicle queue ahead. In this context, to realize a more practical control system, this paper proposes an enhanced EAD strategy of connected EVs for safely and efficiently driving through a signalized intersection with the consideration of the vehicle queue.

Fig. 4 shows the schematic of the problem, which is singlelane driving, thus the lane-change and overtaking maneuvers are not considered. The ego vehicle is equipped with V2I device

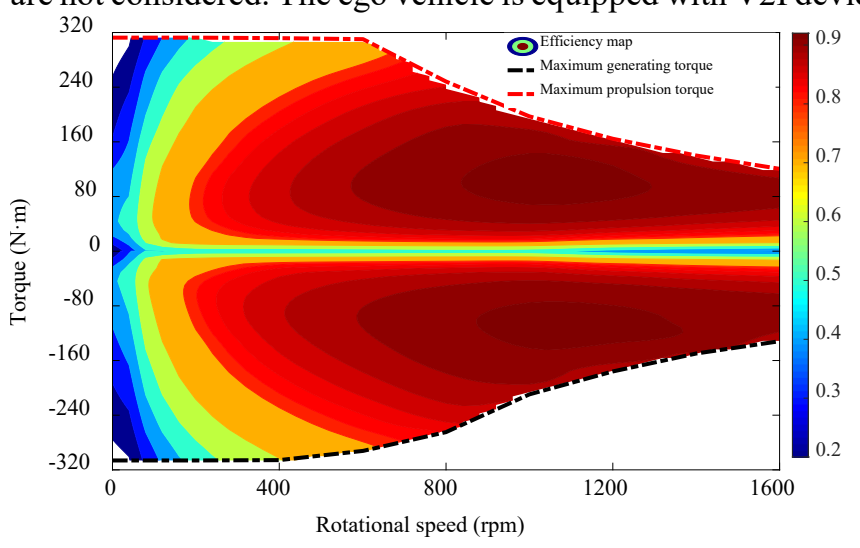

Fig. 2. The efficiency map and maximum torque of IWM.

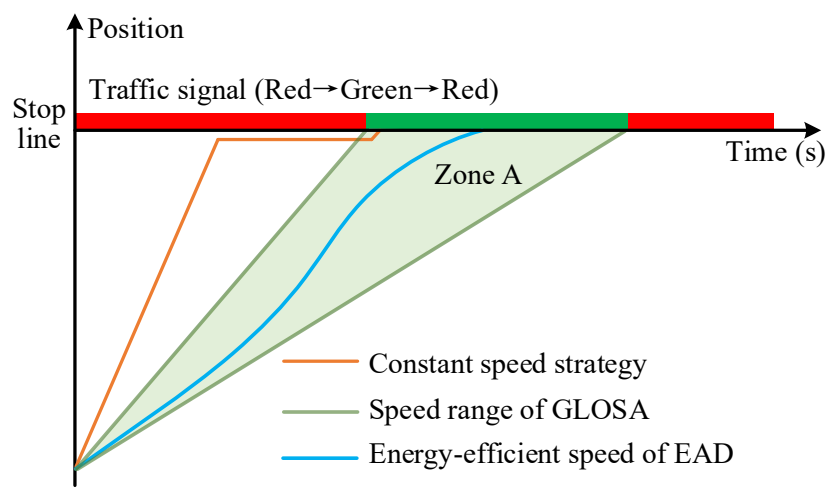

(a)

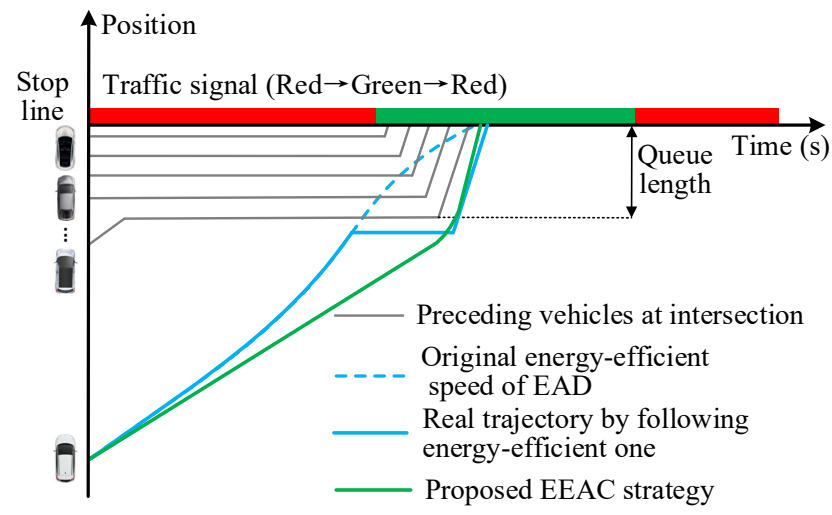

(b)

Fig. 3. The EAD at the signalized intersection. (a) EAD in free-flow traffic. (b) EAD with vehicle queue.

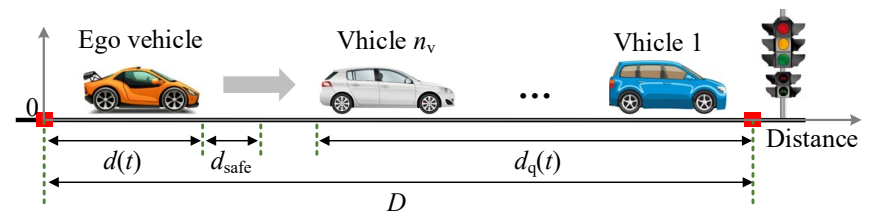

Fig. 4. The schematic of vehicles traveling towards a signalized intersection. 
(Dedicated Short Range Communication or LTE-V) and able to access the traffic information by communicating with the Road Side Unit (RSU) of the intersection within the communication range $D$. Since this paper focuses on the EAD strategy design with the presence of vehicle queue at the intersection, we assume that the traffic signal is a red indication when ego vehicle enters the communication range. The moment that the ego vehicle entering is defined as the initialization of the system $(t=0) . d(t)$ is the travel distance of the ego vehicle, and $d_{q}(t)$ is the distance from the last vehicle of the queue to the stop line. $d_{\text {safe }}$ is a pre-defined minimum inter-vehicle distance, which is used to prevent possible rear-end collision between the ego vehicle and the vehicle in front.

The energy-efficient control problem of the EEAC strategy is formulated as shown in (5) and (6), whose objective is to minimize the total vehicle energy consumption for driving through a signalized intersection.

$$
\underset{\boldsymbol{u} \in \boldsymbol{U}}{\operatorname{Minimize}} J(u(t), x(t))=\int_{0}^{T_{p}} P_{b} d t
$$

s.t

$$
\begin{gathered}
d(0)=0, v(0)=v_{s} \\
d\left(T_{p}\right)=D, v\left(T_{p}\right)=v_{\text {des }} \\
{[D-d(t)]-d_{q}(t) \geq d_{\text {safe }}} \\
u_{\min } \leq u(t) \leq u_{\max } \\
v_{\min } \leq v(t) \leq v_{\max } \\
a_{\min } \leq a(t) \leq a_{\max } \\
\dot{a}(t) \leq a_{J} \\
\dot{x}(t)=f(u(t), x(t))
\end{gathered}
$$

where $T_{p}$ is the moment when the ego vehicle drives through the stop line, which is determined by the predicted vehicle queue discharged time $T_{q}$, i.e., $T_{p}=T_{q}$. This constraint is intended to improve traffic throughput. Operational constraints are introduced in (6) to ensure overall safe and efficient traffic flow. First, the ego vehicle is expected to catch up with the lead vehicle at the end when $t=T_{p}$. That is, the vehicle needs to drive to the desired distance $D$ at the moment $T_{p}$ with desired speed $v_{\text {des }}$. Second, the ego vehicle also needs to keep a safe distance $d_{\text {safe }}$ to its preceding vehicle, i.e., the last vehicle of the queue. Third, the vehicle should meet the road lowest and highest speed limits, i.e., $v_{\min }$ and $v_{\max }$. Fourth, the ego vehicle acceleration is constrained to meet the driving comfort requirement. On one hand, the acceleration $a$ is subject to driving comfort constraints, i.e., the maximum braking deceleration $a_{\min }<0$ and driving acceleration $a_{\max } \geq 0$. On the other hand, the jerk is also limited for ride comfort jerk $a_{J}$. Finally, the control force is subject to physical limits $u_{\text {min }}<0$ and $u_{\max } \geq 0$, which are the maximum propulsion and braking force, respectively.

\section{Control Framework}

The whole control framework for solving the optimal control problem in (5) and (6) is shown in Fig. 5, which is composed of queue discharge prediction, long-term optimization, and local adaptation. The queue discharge prediction part predicts the discharge time of the vehicle queue after the traffic signal turns to green indication, which is treated as the first constraint in (6) as mentioned in the last subsection. DP is used in the upperstage to solve the energy-efficient control problem and derive the globally optimized control trajectories.

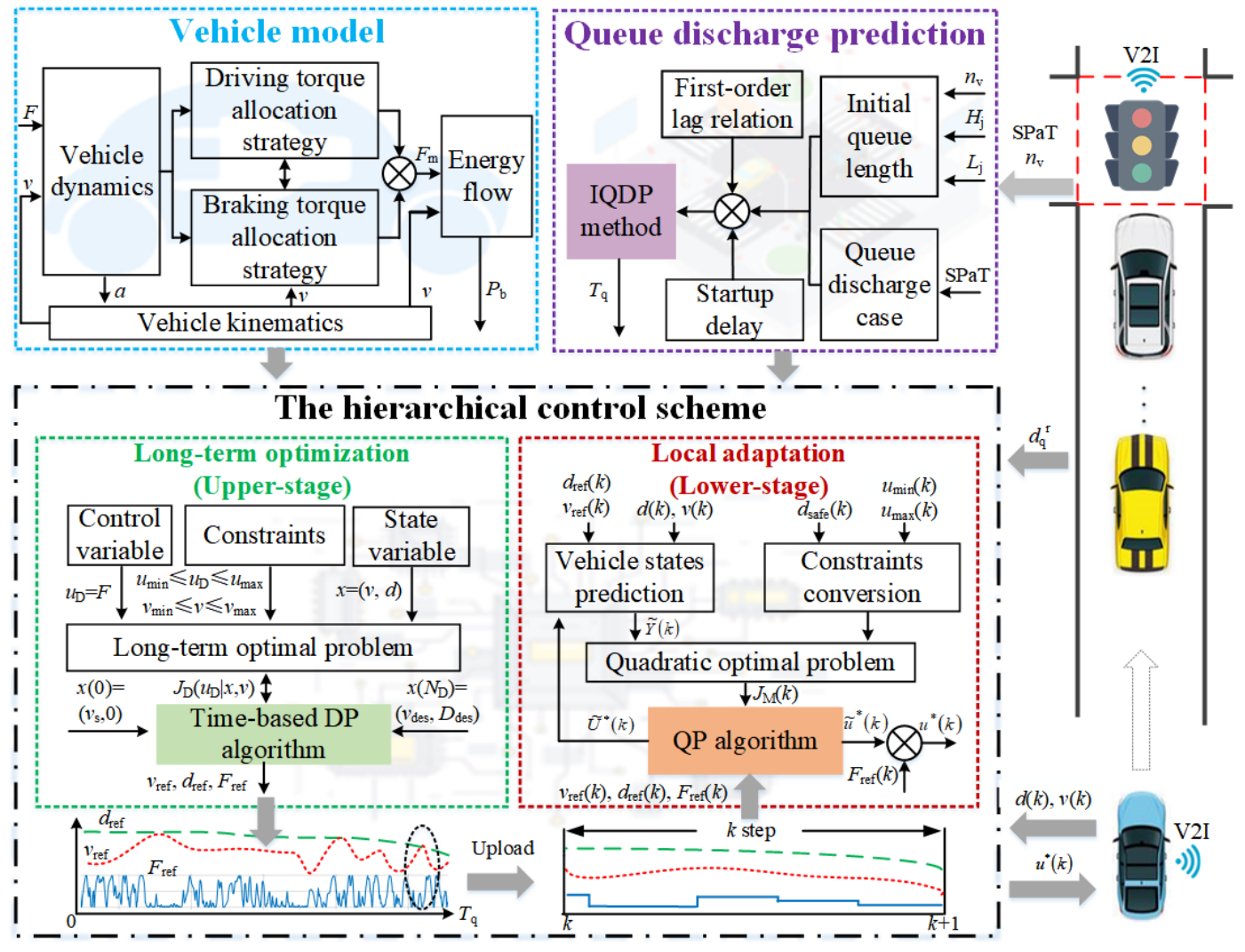

Fig. 5. The framework of the EEAC strategy. 
However, the DP cannot account for unforeseen disturbances, for example, the prediction errors. Rear-end collision may occur if we just follow the derived optimal control rules. Thus, in the lower-stage, an online LTV-MPC is proposed to follow the optimal speed derived from the upper-stage over a smaller time step while accounting for unforeseen disturbances.

\section{Queue Discharge Prediction AT InTERSECTION}

This section will formulate a vehicle queue discharge prediction method which is used for predicting the time of queue dispersion at the intersection. The ego vehicle will communicate with the RSU to access the SPaT and speed limits of the road. It is assumed that all vehicles can pass through the intersection within a green signal, thus, only the traffic signal transition time $t_{s}$ (from red to green) is required. Since not every vehicle is equipped with a V2I device, the ego vehicle cannot get access to the locations and speeds of the vehicles in the queue. However, the RSU will provide the number of vehicles for the queue $\left(n_{v}\right)$ by using in-ground loop detectors [37]. To achieve accurate detection at the intersection segment, the in-ground loop detectors are deployed at both the entrance and stop line of the intersection segment.

As shown in Fig. 4, supposing that the vehicle queue at the intersection is composed of $n_{v}$ heterogeneous vehicles, which are indexed from 1 to $n_{v}$. Then, the initial distance from the rear end of the $n_{v}$ th vehicle to the stop line is defined as $d_{n_{v}}(0)$ and calculated by (7),

$$
d_{n_{v}}(0)=\sum_{j=1}^{n_{v}}\left(L_{j}+H_{j}\right)
$$

where $H_{j}$ is the standstill spacing of the $j$ th vehicle, and $H_{1}$ represents the space between the first vehicle and the stop line. $L_{j}$ is the length of the $j$ th vehicle. Thus, the maximum queue length occurs at the moment $t=0$, which is equal to the distance of the $n_{v}$ th vehicle to stop line, i.e., $d_{q}(0)=d_{n_{v}}(0)$. The movement of the preceding vehicles is predicted through

$$
\dot{v}_{J}(t)=a_{j}(t)
$$

For computational efficiency of the prediction, we simply assume that all the vehicles in the queue follow an identical constant acceleration (until $v_{\max }$ is reached), when the traffic signal turns to green indication. A first-order lag relation is introduced to represent the dynamic response performance, i.e.,

$$
\tau_{j} \dot{a}_{j}(t)+a_{j}(t)=a_{d}
$$

where $a_{d}$ is the desired acceleration, which equates to half of the maximum comfort acceleration. $\tau_{j}$ is the time constant of the response. In addition, a delay coefficient $\kappa_{j}$ is introduced to represent the delay caused by the response of the driver when the vehicle is starting.

Thus, the position of each vehicle in the queue is calculated from two cases. When the traffic signal is red or its preceding vehicle have not started (i.e., $t<t_{s}+\sum_{j=1}^{n_{v}} \kappa_{j}$ ), the $n_{v}$ th vehicle is keeping standing, i.e.,

$$
d_{n_{v}}(t)=d_{n_{v}}(0)
$$

Otherwise, the distance of the $n_{v}$ th vehicle to the stop line is calculated by (11),

$$
d_{n_{v}}(t)=d_{n_{v}}(0)-\int v_{n_{v}}(t) d t
$$

Thus, the vehicle queue length $d_{q}(t)$ is determined by the movement of the final $n_{v}$ th vehicle, that is $d_{q}(t)=d_{n_{v}}(t)$. Besides, when the ego vehicle has not passed through the intersection (i.e., $d_{n_{v}}(t) \leq d_{n_{v}}(0)$ ), the duration of the $n_{v}$ th vehicle $T_{n_{v}}$ approaching the stop line can be evaluated by (12),

$T_{n_{v}}=$

$$
\left\{\begin{array}{cc}
t_{s}+\sum_{j=1}^{n_{v}} \kappa_{j}+\frac{\sqrt{2 a_{d} d_{n_{v}}(0)}}{a_{d}} & \text { if } d_{n_{v}}(0) \leq \frac{v_{\max }^{2}}{2 a_{d}} \\
t_{s}+\sum_{j=1}^{n_{v}} \kappa_{j}+\frac{v_{\max }}{a_{d}}+\frac{d_{n_{v}}(0)-\frac{v_{\max }^{2}}{2 a_{d}}}{v_{\max }} & \text { otherwise }
\end{array}\right.
$$

Finally, the anticipated duration of the vehicle queue approaching the stop line $T_{q}$ is determined by the last $n_{v}$ th vehicle, i.e., $T_{q}=T_{n_{v}}$. The predicted vehicle queue discharge time $T_{q}$ is a fixed value, which will be utilized to determine the total number of time steps in the upper-stage of EEAC strategy. The predicted pass through speed of the last vehicle in the queue is also used as the desired speed $v_{\text {des }}$ in the upper-stage optimization.

\section{THE HIERARCHICAL EEAC STRATEGY}

To generate the energy-efficient speed trajectory, a temporaldomain optimal control problem is formulated in Section II. This section will present the hierarchical EEAC strategy, where the upper-stage uses DP to calculate the global optimal control for long-term energy-efficient driving and the lower-stage deploys LTV-MPC to derive the feasible actions for safe local adaptation.

\section{A. Long-Term Optimization}

The upper-stage of the EEAC strategy uses DP to optimize the trajectory of vehicle traction force. Since DP calculates the optimal solutions by breaking the problem into simpler subproblems, the whole optimization problem in (5) is discretized in the time-domain and rewritten in a discrete form with the time step $\Delta t_{D}$. Since the time step is small, the vehicle traction force of each sub-problem is assumed to be constant.

The states and control variables of the optimal problem should be determined first. As modeled in Section II, the vehicle travel distance and speed are selected as the state $x$. The vehicle control force is selected as the control variable, and denoted by $u=\left[u(0) \cdots u\left(N_{D}-1\right)\right]^{T} . N_{D}$ is the total number of time steps, which is determined by the queue discharge time $T_{q}$, i.e., $N_{D}=\frac{T_{q}}{\Delta t_{D}}+1$. Besides, the state and control variables are quantized for DP calculation as shown in Table I. 
TABLE I

GRIDS OF STATE AND CONTROL VARIABLES

\begin{tabular}{ccc}
\hline \hline & Variable & Grid \\
\hline \multirow{2}{*}{ State } & Speed $v\left(m \cdot s^{-1}\right)$ & {$\left[v_{\min }: 0.1: v_{\text {max }}\right]$} \\
& Travel distance $d(m)$ & {$[0: 0.2: D]$} \\
Control & Traction force $u(N)$ & {$\left[u_{\text {min }}: 15: u_{\text {max }}\right]$} \\
\hline \hline
\end{tabular}

Thus, the global optimal problem is indexed by $k$ as shown in (13),

$\underset{u_{D} \in \boldsymbol{U}}{\operatorname{Minimize}} J_{D}\left(u_{D}, x\right)=$
$\sum_{k=1}^{N_{D}} P_{b}(k) \Delta t_{D}+\alpha_{1}\left(v\left(N_{D}\right)-v_{\text {des }}\right)^{2}+\alpha_{2}\left(d\left(N_{D}\right)-D\right)^{2}$

s.t.

$$
\begin{gathered}
u_{\min }(k) \leq u_{D}(k) \leq u_{\max }(k) \\
v_{\min }(k) \leq v(k) \leq v_{\max }(k) \\
\dot{x}(k)=f(u(k), x(k))
\end{gathered}
$$

Even we made the constraints of the terminal state in (6) for improving traffic throughput, the terminal conditions of the distance and velocity have been relaxed by converting them to soft constraints and merged into the objective function as (13), with $\alpha_{1}$ and $\alpha_{2}$ two weighting factors for the latter two terms. In addition, denote the transitional cost-to-go function from $k$ step to $k+1$ step $L\left(u_{D}(k), x(k)\right)$ and the penalty function for the terminal states $G_{N}\left(x\left(N_{D}\right)\right)$, i.e.,

$$
\begin{gathered}
L\left(u_{D}(k), x(k)\right)=P_{b}(k) \Delta t_{D} \\
G_{N}\left(x\left(N_{D}\right)\right)=\alpha_{1}\left(v\left(N_{D}\right)-v_{d e s}\right)^{2}+\alpha_{2}\left(d\left(N_{D}\right)-D\right)^{2}
\end{gathered}
$$

The formulated global optimal problem is solved recursively by following the Bellman Principle [38].

$N_{D}-1$ step:

$$
\begin{aligned}
J_{N_{D}-1}^{*}\left(x\left(N_{D}-1\right)\right)= \\
\quad \min _{u_{D} \in \boldsymbol{U}}\left[L\left(u_{D}\left(N_{D}-1\right), x\left(N_{D}-1\right)\right)+G_{N}\left(x\left(N_{D}\right)\right)\right]
\end{aligned}
$$

$k$ step:

$$
J_{k}^{*}(x(k))=\min _{u_{D} \in \boldsymbol{U}}\left[L\left(u_{D}(k), x(k)\right)+J_{k+1}^{*}(x(k+1))\right]
$$

where $J_{k}^{*}(x(k))$ is the minimum cost-to-go value at the moment $k$. Through the backward calculation, the optimal control law can be obtained.

\section{B. Local Adaptation}

If no disturbances emerge, the ego vehicle could achieve efficient and safe driving by following the optimal solution derived in the upper-stage. However, the reference speed cannot be perfectly tracked, and the prediction error for the queue discharge time is inevitable. The rear-end collision may occur if implementing the optimal control solution directly. Thus, the lower-stage controller aims to develop an MPC-based controller for the ego vehicle to track the optimal vehicle speed trajectory while keeping a safe distance to its preceding vehicle.
Equation (18) shows the control problem formulation of MPC for the local adaptation. The first term is to minimize the vehicle speed and position tracking errors, and the second term considers the vehicle driving comfort by minimizing the increment of the traction forces.

$$
\begin{aligned}
\underset{u_{M} \in \boldsymbol{U}}{\operatorname{Minimize}} & J_{M}\left(u_{M} \mid k\right)= \\
& \sum_{i=1}^{N_{P}}\|\tilde{x}(k+i \mid k)\|_{Q}^{2}+\sum_{j=1}^{N_{C^{-}}-1}\|\Delta u(k+j \mid k)\|_{R}^{2}
\end{aligned}
$$

s.t.

$$
\begin{gathered}
u_{\min }(k+j) \leq u_{M}(k+j) \leq u_{\max }(k+j) \\
{[D-d(k+j)]-d_{q}^{r}(k+j) \geq d_{\text {safe }}(k+j)} \\
\dot{x}(k+j)=f(u(k+j), x(k+j))
\end{gathered}
$$

where $\tilde{x}$ is the tracking error for ego vehicle, i.e., $\tilde{x}=$ $\left[d-d_{\text {ref }} v-v_{\text {ref }}\right]^{T} . d_{\text {ref }}$ and $v_{\text {ref }}$ are the reference vehicle position and speed trajectory, respectively, which are derived from the upper-stage long-term optimization. $N_{p}$ and $N_{c}$ are the prediction and control horizon for the MPC, respectively. $d_{q}^{r}$ is the actual vehicle queue length, $\left(u_{M} \mid k\right)$ represents the derived control variable of MPC at $k$ moment, $Q$ and $R$ are the weighting factor matrix.

In addition, a proper inter-vehicle distance between the ego vehicle and its preceding is designed to avoid the possible rearend collision [39] and defined in (19),

$$
d_{\text {safe }}(k+j)=H_{0}+\lambda\left[v(k+j) \Delta t_{M}+\frac{v^{2}(k+j)}{2\left|a_{\text {bmax }}\right|}\right]
$$

where the first term $H_{0}$ is a "static gap" and determines the minimum gap when the vehicle is stopped, and the second term is a "dynamic gap" providing an extra safe gap with increased speed. $\lambda \geq 1$ is a margin for driving safety concerns. $a_{b m a x}$ is the vehicle maximum braking deceleration, $\Delta t_{M}$ is the time step of MPC controller, and $v_{p}(k)$ is the speed of the preceding vehicle, which is measured by the equipped radar in the ego vehicle. It should be noted that the safety distance is generally less than the detectable distance of the vehicle radar, thus the rear-end collision avoidance control is enabled when the preceding vehicle is detected.

The MPC problem in (18) is solved in three steps:

\section{1) State Prediction}

To reduce the computational burden, we employ the approximate linearization method to transform the nonlinear time-varying vehicle longitudinal dynamics to a linear timevarying (LTV) system [40]. Then, the vehicle state error model is obtained,

$$
\dot{\tilde{x}}(t)=\left[\begin{array}{cc}
0 & 1 \\
0 & -\frac{C_{D} A \rho v_{r e f}(t)}{m}
\end{array}\right] \tilde{x}(t)+\left[\begin{array}{l}
0 \\
\frac{1}{m}
\end{array}\right] \tilde{u}(t)
$$

where $\tilde{u}(t)=F(t)-F_{\text {ref }}(t), F_{\text {ref }}$ is the reference vehicle control force which is derived from the upper-stage optimization. The LTV system (20) is discretized by the Euler method, yielding 


$$
\tilde{x}(k+1)=
$$

$$
\left[\begin{array}{cc}
1 & \Delta t_{M} \\
0 & 1-\frac{C_{D} A \rho v_{r e f}(k) \Delta t_{M}}{m}
\end{array}\right] \tilde{x}(k)+\left[\begin{array}{c}
0 \\
\frac{\Delta t_{M}}{m}
\end{array}\right] \tilde{u}(k)
$$

where $\Delta t_{M}$ is the time step. In addition, the system output is defined as the system states, i.e., $\tilde{y}(k+1)=C \tilde{x}(k+1)$, where $C$ is the coefficient matrix.

Denote a state prediction matrix

$$
\varsigma(i \mid k)=\left[\begin{array}{c}
\tilde{x}(i \mid k) \\
\tilde{u}(i-1 \mid k)
\end{array}\right]
$$

where $\tilde{x}(i \mid k)$ represents the system $i$-step-ahead predicted error at the $k$ th step. Substituting (21) into (22), the state-space model for state prediction is obtained

$$
\tilde{Y}(k)=\Gamma_{k} \varsigma(k)+\Xi_{k} \Delta \widetilde{U}(k)
$$

with

$$
\begin{aligned}
& \tilde{Y}(k)=\left[\begin{array}{lllll}
\widetilde{C}_{k, 1} \varsigma(1 \mid k) & \cdots & \widetilde{C}_{k, N_{c}} \varsigma\left(N_{c} \mid k\right) & \cdots & \widetilde{C}_{k, N_{p}} \varsigma\left(N_{p} \mid k\right)
\end{array}\right]_{1 \times N_{p}}^{T} \\
& \Gamma_{k}=\left[\begin{array}{lllllll}
\tilde{C}_{k, i} \tilde{A}_{k, i} & \cdots & \tilde{C}_{k, i} \tilde{A}_{k, i}^{N_{c}} & \cdots & \tilde{C}_{k, i} \tilde{A}_{k, i}^{N_{p}}
\end{array}\right]_{1 \times N_{p}}^{T} \\
& \Xi_{k}=\left[\begin{array}{cccc}
\tilde{C}_{k, i} \tilde{B}_{k, i} & 0 & \cdots & 0 \\
\tilde{C}_{k, i} \tilde{A}_{k, i} \tilde{B}_{k, i} & \tilde{C}_{k, i} \tilde{B}_{k, i} & \cdots & 0 \\
\vdots & \vdots & \ddots & \vdots \\
\tilde{C}_{k, i} \tilde{A}_{k, i}^{N_{c}} \tilde{B}_{k, i} & \tilde{C}_{k, i} \tilde{A}_{k, i}^{N_{c}-1} \tilde{B}_{k, i} & \cdots & \tilde{C}_{k, i} \tilde{A}_{k, i} \tilde{B}_{k, i} \\
\vdots & \vdots & \ddots & \vdots \\
\tilde{C}_{k, i} \tilde{A}_{k, i}^{N_{p}-1} \tilde{B}_{k, i} & \tilde{C}_{k, i} \tilde{A}_{k, i}^{N_{p}-2} \tilde{B}_{k, i} & \cdots & \tilde{C}_{k, i} \tilde{A}_{k, i}^{N_{p}-N_{c}-1} \tilde{B}_{k, i}
\end{array}\right]_{N_{p} \times N_{c}} \\
& \Delta \widetilde{U}(k)=\left[\begin{array}{llll}
\Delta \tilde{u}(k \mid k) & \Delta \tilde{u}(k+1 \mid k) & \cdots & \Delta \tilde{u}\left(k+N_{c} \mid k\right)
\end{array}\right]_{1 \times N_{c}}^{T} \\
& \tilde{A}_{k, i}=\left[\begin{array}{ccc}
1 & \Delta t_{M} & 0 \\
0 & 1-\frac{C_{D} A \rho v_{r e f}(i \mid k) \Delta t_{M}}{m} & \frac{\Delta t_{M}}{m} \\
0 & 0 & 1
\end{array}\right] \\
& \tilde{B}_{k, i}=\left[\begin{array}{lll}
0 & \frac{\Delta t_{M}}{m} & 1
\end{array}\right]^{T}, \tilde{C}_{k, i}=\left[\begin{array}{lll}
1 & 0 & 0 \\
0 & 1 & 0
\end{array}\right]
\end{aligned}
$$

where $\tilde{Y}(k)$ and $\Delta \widetilde{U}(k)$ are output and control increments of the prediction model, respectively. $\varsigma(k)$ is the state variable at the $k$ th step. Besides, since $N_{p} \geq N_{c}$, the control variable beyond $N_{c}$ is always the last control variable of $N_{p}$.

2) Constraints Conversion

The solution of the proposed LTV-MPC is the control increment. Thus, the subsection will convert the constraints to the form of control increment.

For the first constraint in (18) is converted to

$$
U_{\min } \leq U_{M}(k) \leq U_{\max }
$$

with

$$
\begin{aligned}
& U_{M}(k)=\left[\begin{array}{ccc}
1 & \cdots & 0 \\
\cdots & \ddots & \cdots \\
1 & \cdots & 1
\end{array}\right]_{N_{c} \times N_{c}} \Delta \widetilde{U}(k)+1_{N_{c}} u_{M}(k-1) \\
& U_{\min }=\left[\begin{array}{llll}
u_{\min }(k) & u_{\min }(k+1) & \cdots & u_{\min }\left(k+N_{c}-1\right)
\end{array}\right]_{1 \times N_{c}}^{T} \\
& U_{\max }=\left[\begin{array}{llll}
u_{\max }(k) & u_{\max }(k+1) & \cdots & u_{\max }\left(k+N_{c}-1\right)
\end{array}\right]_{1 \times N_{c}}^{T}
\end{aligned}
$$

where $1_{N_{c}}$ is a column vector with $N_{c}$ rows. $U_{\min }$ and $U_{\max }$ are the minimum and maximum value set of control variable in $N_{c}$. Moreover, in the prediction horizon, the relation between the control variable and travel distance is

$$
\begin{aligned}
& d(k+i)= \\
& \quad d(k+i-1)+v(k+i-1) \Delta t_{M}+0.5 a(k+i) \Delta t_{M}^{2}
\end{aligned}
$$

where $a$ is the vehicle acceleration, which is calculated by (26)

$$
a(k+i)=\frac{u_{M}(k+i)-C_{r}(k+i-1)}{m}
$$

where $C_{r}$ is the sum of resistance, i.e., rolling resistance, gravity resistance, and aerodynamic drag, $C_{r}(k)=m g f \cos \theta+$ $m g \sin \theta+0.5 C_{D} A \rho v^{2}(k)$.

Combining the (19), (25), and (26), the collision avoidance constraint is converted to

$$
\begin{gathered}
D_{\text {safe }} \geq\left[\begin{array}{ccc}
\frac{\left(\Delta t_{M}\right)^{2}}{m} & \cdots & 0 \\
\vdots & \ddots & \vdots \\
0 & \cdots & \left.\frac{\left(N_{c} \Delta t_{M}\right)^{2}}{m}\right]_{N_{c} \times N_{c}} U_{M}(k)+ \\
d(k-1)-v(k-1) \Delta t_{M}-\frac{C_{r}(k-1)}{m} \Delta t_{M}^{2} \\
d(k-1)-v(k-1) 2 \Delta t_{M}-\frac{C_{r}(k-1)}{m}\left(2 \Delta t_{M}\right)^{2} \\
\vdots \\
d(k-1)-v(k-1) N_{c} \Delta t_{M}-\frac{C_{r}(k-1)}{m}\left(N_{c} \Delta t_{M}\right)^{2}
\end{array}\right]_{N_{c} \times 1}
\end{gathered}
$$

with

$$
D_{\text {safe }}=D+d_{q}(k)-\left[d_{\text {safe }}(k) \quad \cdots \quad d_{\text {safe }}\left(k+N_{c}-1\right)\right]_{1 \times N_{c}}^{T}
$$

3) LTV-MPC Solving

In this paper, the local adaptation control problem in (18) is solved using quadratic programming $(\mathrm{QP})$, and the objective function and constraints are both converted to the standard QP form [41]. Then, the control problem is converted to

$$
\begin{aligned}
& J_{M}(\Delta \widetilde{U} \mid k)= \\
& \frac{1}{2}\left[\begin{array}{cc}
\Delta \widetilde{U}(k)^{T} \\
0
\end{array}\right]\left[\begin{array}{cc}
2\left(\Xi_{k}^{T} Q \Xi_{k}+R\right) & 0 \\
0 & 0
\end{array}\right]\left[\begin{array}{c}
\Delta \widetilde{U}(k)^{T} \\
0
\end{array}\right]^{T}\left[\begin{array}{c}
2 E_{k}^{T} Q \Xi_{k} \\
0
\end{array}\right]^{T}\left[\begin{array}{c}
\Delta \widetilde{U}(k)^{T} \\
0
\end{array}\right]^{T}
\end{aligned}
$$

s.t

$$
\begin{aligned}
& U_{\min } \leq\left[\begin{array}{ccc}
1 & \cdots & 0 \\
\cdots & \ddots & \cdots \\
1 & \cdots & 1
\end{array}\right]_{N_{c} \times N_{c}} \Delta(0)=\left[\begin{array}{ll}
0 & v_{S}
\end{array}\right]^{T} \\
& D_{\text {safe }} \geq\left[\begin{array}{ccc}
\frac{\left(\Delta t_{M}\right)^{2}}{m} & \cdots & 0 \\
\vdots & \ddots & \vdots \\
0 & \cdots & \frac{\left(N_{c} \Delta t_{M}\right)^{2}}{m}
\end{array}\right]_{N_{c} \times N_{c}} U_{M}(k)+ \\
& {\left[\begin{array}{c}
d(k-1)-v(k-1) \Delta t_{M}-\frac{C_{r}(k-1)}{2 m} \Delta t_{M}^{2} \\
d(k-1)-v(k-1) 2 \Delta t_{M}-\frac{C_{r}(k-1)}{2 m}\left(2 \Delta t_{M}\right)^{2} \\
\vdots \\
d(k-1)-v(k-1) N_{c} \Delta t_{M}-\frac{C_{r}(k-1)}{2 m}\left(N_{c} \Delta t_{M}\right)^{2}
\end{array}\right]_{N_{c} \times 1}}
\end{aligned}
$$

A series of control variable increments $\Delta \widetilde{U}_{k}^{*}$ within the control horizon $N_{c}$ can be obtained by solving the QP problem in every control period. Then, the resulted optimal control variable for the first step of the prediction horizon $N_{P}$ is

$$
\tilde{u}^{*}(k)=\tilde{u}(k-1)+\left[\begin{array}{llll}
I_{N_{c}} & 0 & \cdots & 0
\end{array}\right]_{1 \times N_{c}} \Delta \widetilde{U}_{k}^{*}
$$

Finally, the vehicle traction force is

$$
u^{*}(k)=\tilde{u}^{*}(k)+F_{\text {ref }}(k)
$$

where $F_{\text {ref }}$ are the reference vehicle control force which is 
derived from the upper-stage optimization.

\section{Simulation AND RESUlTS}

To evaluate the performance of the proposed EEAC strategy, we conduct several simulations by using an open-source microscopic multi-modal traffic simulation, SUMO, on a PC with Intel Core i7-8700@3.20GHz CPU and 16GB RAM.

\section{A. Simulation Setup}

In SUMO, we built the road section map using OpenStreetMap, and the Intelligent Diver Model (IDM) is employed as the car-following model to simulate the queue movement dynamics at an intersection. The IDM is a wellaccepted model for single-lane traffic flow, it can be used to describe the dynamic behavior of human-driven or autonomous vehicles [42]. It can be described by

$$
\left\{\begin{array}{c}
\dot{d}_{j}=v_{j} \\
\dot{v}_{j}=a_{\max }\left[1-\left(\frac{v_{j}}{v^{*}}\right)^{\varpi}-\left(\frac{d^{*}}{\widetilde{d_{j}}}\right)^{2}\right]
\end{array}\right.
$$

with

$$
\left.d^{*}=H_{j}+T_{h, j} v_{j}+\frac{v_{j} \widetilde{v}_{J}}{2 \sqrt{a_{\min } a_{\max }}}\right\}
$$

where $\widetilde{d}_{j}$ is the bumper-to-bumper inter-vehicle clearance of the $j$ th vehicle and preceding one, $\widetilde{v}_{j}$ is the velocity difference of the $j$ th vehicle and preceding one. $d^{*}$ and $v^{*}$ are the desired inter-vehicle clearance and road speed limit, respectively. $T_{h, j}$ is the $j$ th vehicle safe headway, $\varpi$ is acceleration exponent. In this paper, we evaluate the proposed EEAC strategy in a stochastic traffic environment, thus the parameters $H_{j}, T_{h, j}$, $a_{\min }$, and $a_{\max }$ are initialized randomly. Table II shows the parameter bounds used for the IDM model implemented in the stochastic traffic environment $[43,44]$.

TABLE II

PARAMETER BOUNDS

\begin{tabular}{cc}
\hline \hline Parameter & Bounds \\
\hline Standstill spacing $H_{j}$ & {$[1 \mathrm{~m}, 3 \mathrm{~m}]$} \\
Safe time headway $T_{h, j}$ & {$[0.5 \mathrm{~s}, 2 \mathrm{~s}]$} \\
Maximum comfort acceleration $a_{\max }$ & {$\left[2 \mathrm{~m} \cdot \mathrm{s}^{-2}, 4 \mathrm{~m} \cdot \mathrm{s}^{-2}\right]$} \\
Maximum comfort deceleration $a_{\min }$ & {$\left[-2 \mathrm{~m} \cdot \mathrm{s}^{-2},-4 \mathrm{~m} \cdot \mathrm{s}^{-2}\right]$} \\
Acceleration exponent $\varpi$ & 4 \\
\hline \hline
\end{tabular}

Table III lists the main parameters of the ego vehicle in this paper. The time step $\Delta t$ is $0.1 \mathrm{~s}$ for DP algorithm in long-term optimization. The weighting factors $\alpha_{1}$ and $\alpha_{2}$ in upper-stage optimization are both 800 , and weighting matrices in (18) are $Q$ $=100 I_{2 N_{p} \times 2 N_{p}}$ and $R=0.1 I_{N_{c}}$. In addition, the prediction and control horizon and the step size of the MPC are set to $N_{p}=100$ and $\Delta t_{M}=0.01$, which finds a good compromise between optimality and computational efficiency, as verified by a systematic simulative analysis for various step sizes and horizon lengths. Two simulations with various traffic parameters were conducted, which are referred to as Scenarios $\mathrm{A}$ and $\mathrm{B}$ as listed in Table IV.
TABLE III

\begin{tabular}{|c|c|c|c|}
\hline Component & \multicolumn{2}{|c|}{ Parameter } & Value \\
\hline \multicolumn{3}{|c|}{ Maximum power } & $20.75 \mathrm{~kW} /-20.53 \mathrm{~kW}$ \\
\hline \multirow[t]{2}{*}{ Motor } & \multicolumn{2}{|c|}{ Maximum torque } & $12.50 \mathrm{Nm} /-311.50 \mathrm{Nm}$ \\
\hline & \multicolumn{2}{|c|}{ Maximum speed } & $1600 \mathrm{rpm}$ \\
\hline \multirow{3}{*}{ Li-ion Battery } & \multicolumn{2}{|c|}{ Capacity } & $140 \mathrm{Ah}$ \\
\hline & \multicolumn{2}{|c|}{ Open circuit voltage } & $360 \mathrm{~V}$ \\
\hline & \multicolumn{2}{|c|}{ Efficiency $\eta_{b}$} & 0.9 \\
\hline & \multicolumn{2}{|c|}{ Mass $m$} & $1421 \mathrm{~kg}$ \\
\hline & \multicolumn{2}{|c|}{ Front area $A$} & $2.22 \mathrm{~m}^{2}$ \\
\hline & \multicolumn{2}{|c|}{ Aerodynamic drag coefficient $C_{D}$} & 0.3 \\
\hline & \multicolumn{2}{|c|}{ Air density $\rho$} & $1.206 \mathrm{~kg} \cdot \mathrm{m}^{-3}$ \\
\hline Vabial & \multicolumn{2}{|c|}{ Rotational inertia coefficient $\delta$} & 1.022 \\
\hline रूता & \multicolumn{2}{|c|}{ Rolling resistance coefficient $f$} & 0.015 \\
\hline & \multicolumn{2}{|c|}{ Tire radius $r_{w}$} & $0.325 \mathrm{~m}$ \\
\hline & \multicolumn{2}{|c|}{ Road gradient $\theta$} & 0 \\
\hline & \multicolumn{2}{|c|}{ Auxiliary power $P_{a}$} & $300 \mathrm{~W}$ \\
\hline & \multicolumn{2}{|c|}{ Acceleration of gravity $g$} & $9.8 \mathrm{~m} \cdot \mathrm{s}^{-2}$ \\
\hline \multicolumn{4}{|c|}{$\begin{array}{c}\text { TABLE IV } \\
\text { SIMULATION SCENARIOS } \\
\end{array}$} \\
\hline \multicolumn{2}{|c|}{ Parameter } & Scenario A & Scenario B \\
\hline \multicolumn{2}{|c|}{ Vehicles of queue $n_{\mathrm{v}}$} & 10 & 5 \\
\hline \multicolumn{2}{|c|}{ Switching time $t_{\mathrm{s}}$} & $28 \mathrm{~s}$ & $23 \mathrm{~s}$ \\
\hline \multicolumn{2}{|c|}{ Communication range $D$} & \multicolumn{2}{|r|}{$350 \mathrm{~m}$} \\
\hline \multicolumn{2}{|c|}{ Initial speed $v_{\mathrm{s}}$} & \multicolumn{2}{|r|}{$54 \mathrm{~km} \cdot \mathrm{h}^{-1}$} \\
\hline \multicolumn{2}{|c|}{ Minimum speed $v_{\min }$} & \multicolumn{2}{|r|}{$20 \mathrm{~km} \cdot \mathrm{h}^{-1}$} \\
\hline \multicolumn{2}{|c|}{ Maximum speed $v_{\max }$} & \multicolumn{2}{|r|}{$60 \mathrm{~km} \cdot \mathrm{h}^{-1}$} \\
\hline \multicolumn{2}{|c|}{ Fixed signal timing } & & $60 \mathrm{~s}$ \\
\hline
\end{tabular}

VEHICLE PARAMETERS

B. Results and Discussion

Three control strategies are compared in this subsection, i.e., constant speed (CS) [45], regular EAD, and proposed EEAC. For CS strategy, the ego vehicle will approach the intersection at a fixed speed and stop to join the vehicle queue if the traffic signal is a red indication. The braking deceleration is $-3 \mathrm{~m} \cdot \mathrm{s}^{-2}$, and the standstill spacing between the preceding vehicle is $2 \mathrm{~m}$. The regular EAD uses the SPaT information to calculate the eco-friendly speed trajectory to control the vehicle driving through the signalized intersection without stopping as introduced in Section II. In this paper, the regular EAD trajectory is also composed of two stages, where the upperstage calculates the optimal speed trajectory by using DP without considering the vehicle queue, and the lower-stage uses the same LTV-MPC controller as the proposed EEAC to track the derived optimal trajectory.

In the simulation, the time consumption of DP in the upperstage for EEAC strategy is 836.2 seconds and 657.9 seconds of Scenario A and Scenario B, respectively. The average calculation speed of LTV-MPC in each step is 39.2 milliseconds and 38.9 milliseconds of Scenario A and Scenario $\mathrm{B}$, respectively. The local adaption controller (i.e., LTV-MPC) 
has better convergence performance, only requires 0.66 seconds and 0.49 seconds respectively in scenarios $\mathrm{A}$ and $\mathrm{B}$ to reach the steady-state, where the velocity tracking error converges to zero. 1) Energy Efficiency

Figs. 6, 7, and 8 show the travel distance, vehicle speed, and battery power for the CS, regular EAD, and EEAC strategy. Tables V and VI list their average motor efficiency and energy consumption, respectively. The average motor efficiencies of the front and rear axles of different strategies and scenarios are reported in Table $\mathrm{V}$, provided the motor efficiencies are uniform along an axle. In Table VI, the results of the upperstage for regular EAD and EEAC are derived from the longterm optimization controller of both strategies, and the lowerstage results are referred to as the results of the local adaption controller. The sum of battery energy consumption and vehicle kinetic energy changes are combined to calculate the overall energy efficiency improvement to ensure a fair comparison among different strategies.

As observed in Fig. 6, in both Scenarios A and B, the ego vehicle with $\mathrm{CS}$ and regular $\mathrm{EAD}$ has to stop and join the queue before driving through the intersection, while the ego vehicle with EEAC can pass through the intersection without stops due to the availability of the vehicle queue discharge prediction. Fig. 7 shows that the ego vehicle with EEAC strategy decelerates to a lower speed at the beginning, and keeps cruising at this speed for a while in both Scenarios A and B. The reason is that the vehicle tends to consume more energy at high speeds due to the aerodynamic losses. Thus, as shown in Fig. 8, the battery output power is reduced in lower cruising speed than high-speed range. Note that the battery power profiles of the regular EAD and the proposed EEAC involve high-frequency oscillations, which are incurred by the direct implementation of the nonlinear motor efficiency map (see Fig. 2). The results can be smoothened by introducing further approximations (to the motor map or the reference speed generated in the upper-stage), which, however, degrade the optimality of the solution.

In Table $\mathrm{V}$, the EEAC achieves motor efficiency improvement over $20 \%$ and $15 \%$ compared with CS and regular EAD in Scenario A and Scenario B, respectively. It can be concluded that the EEAC strategy can reduce vehicle resistance losses and ensure motor working in a high-efficiency zone. Hence, as shown in Table VI, the energy efficiency is improved. Compared with CS and regular EAD, the EEAC

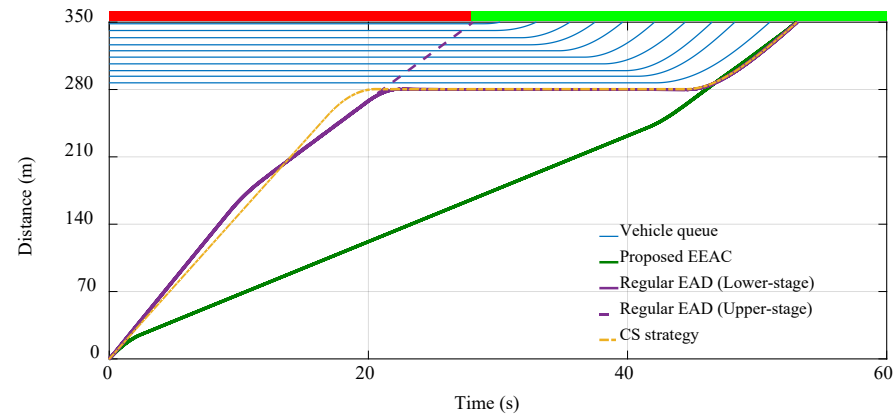

(a) achieves energy consumption reduction by $12.48 \%$ and $12.01 \%$ in Scenario A, and $8.51 \%$ and $11.22 \%$ in Scenario B.

The importance of the queue discharge prediction is also reflected by further comparing the energy consumption of the $\mathrm{CS}$ and regular EAD. Although the anticipated energy saving of the upper-stage of the regular EAD is significantly achieved $19.50 \%$, the actual saving (obtained from the lower-stage) is limited to $0.54 \%$ in Scenario A, and in Scenario B even more energy is consumed than the CS strategy as the upper-stage solution for the regular EAD. The upper-stage optimized trajectory cannot be followed by the lower-stage due to the vehicle queue ahead, which is not modeled and predicted.

In addition, Fig. 9 shows the distance and speed tracking errors for the proposed EEAC. As observed, the velocity trajectory exists dips in the lower-stage at 46.75 seconds and 30.14 seconds in Scenario A and Scenario B, respectively. The reason for this phenomenon is the inevitable prediction error of the upper-stage. As the ego vehicle approaches the preceding vehicle, proper braking is applied to maintain the safe intervehicle distance between the ego and lead vehicles. Even both distance and speed tracking errors are observed, they are small and have a limited impact on energy efficiency as shown in Table VI (with $6.17 \%$ and $4.55 \%$ energy consumption increased). To illuminate the effects of the prediction accuracy, we will conduct more simulations in the next subsection.

2) Influence of Vehicle Queue Discharge Prediction Accuracy on Energy-saving

In this subsection, Scenario A is run 10,000 times in a Monte Carlo type experiment in which the parameters of the vehicle queue were randomized in SUMO, including the acceleration exponent $\varpi$, standstill spacing $H_{\mathrm{j}}$, safe time headway $T_{\mathrm{h}, \mathrm{j}}$, maximum comfort acceleration $a_{\max }$, and maximum comfort deceleration $a_{\min }$ for each vehicle. The feasible set of each parameter is listed in Table II.

To evaluate the prediction accuracy, the vehicle queue discharge time prediction error $\bar{T}_{q}$ is defined in (32),

$$
\bar{T}_{q}=\frac{T_{q}^{r}-T_{q}}{T_{q}} \times 100 \%
$$

where $T_{q}$ is the predicted queue discharge time, and $T_{q}^{r}$ is the actual queue discharge time in the simulations. The prediction error of discharge time is illustrated in Fig. 10.

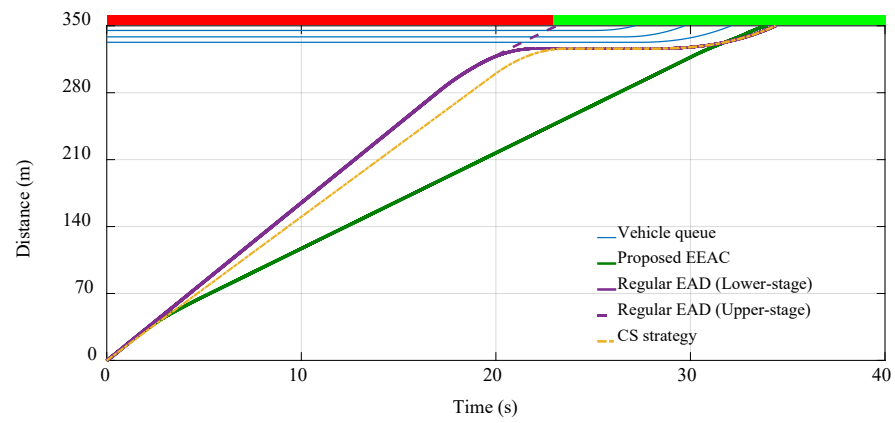

(b)

Fig. 6. The distance trajectory of vehicle queue and ego vehicle. (a) Scenario A. (b) Scenario B. 


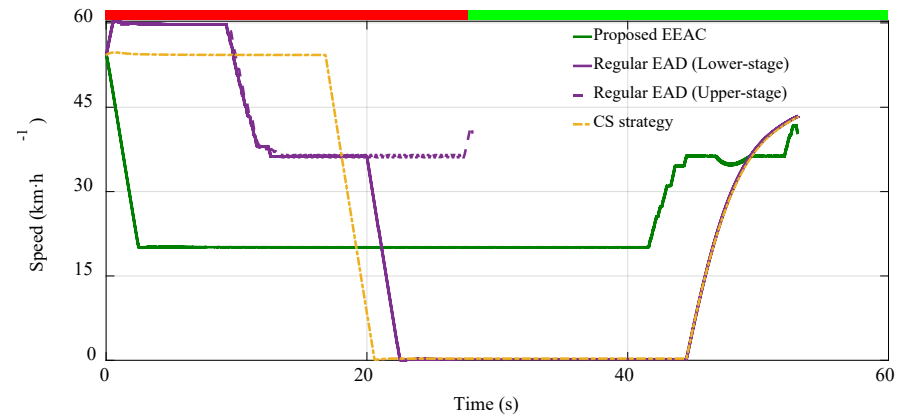

(a)

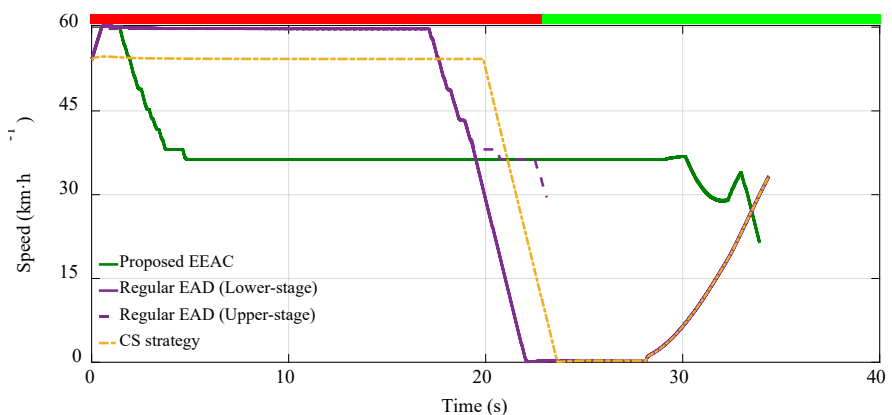

(b)

Fig. 7. The ego vehicle speed trajectory of CS strategy, regular EAD strategy, and EEAC strategy. (a) Scenario A. (b) Scenario B.

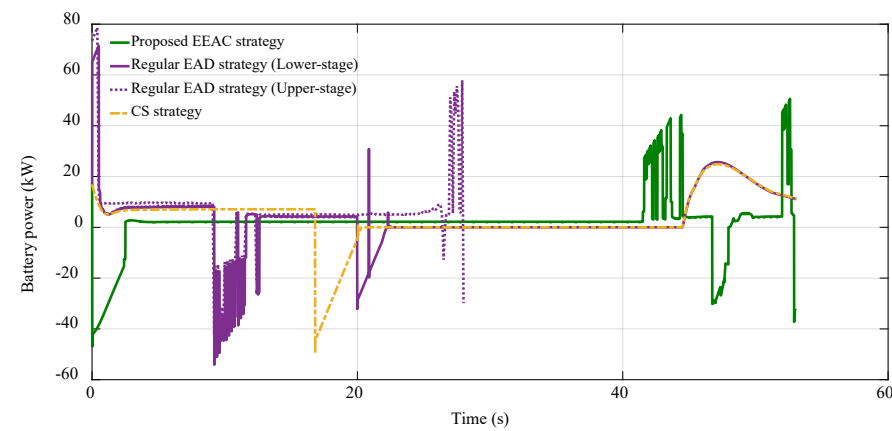

(a)

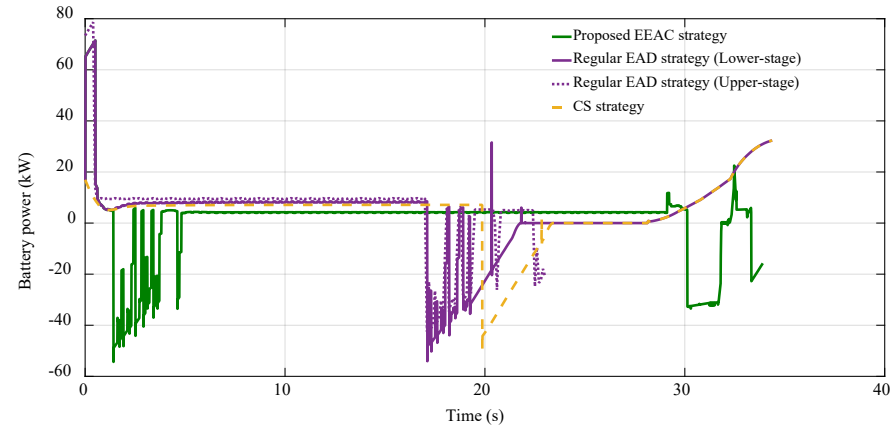

(b)

Fig. 8. The ego vehicle battery power of CS strategy, regular EAD strategy, and EEAC strategy. (a) Scenario A. (b) Scenario B.

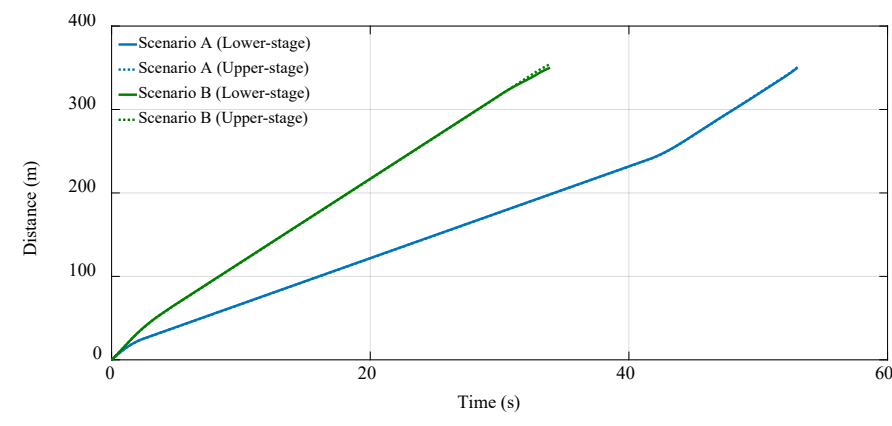

(a)

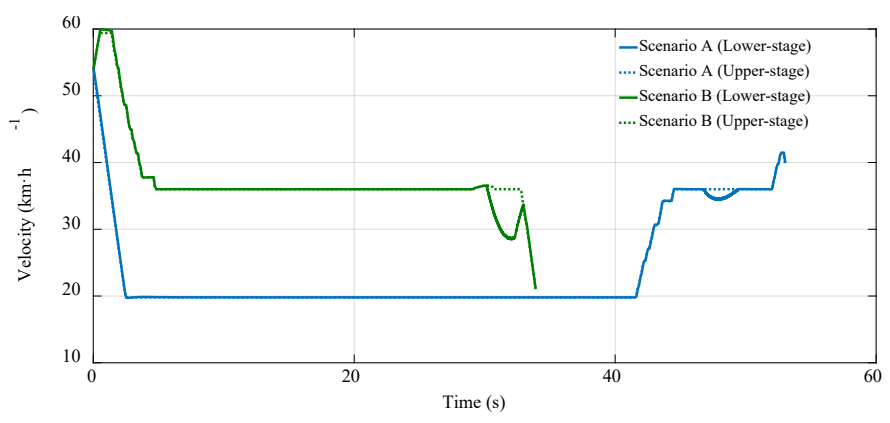

(b)

Fig. 9. The distance and speed trajectory of long-term optimization and local adaption. (a) Distance and the tracking error. (b) Speed and the tracking error.

TABLE V

AVERAGE MOTOR EFFICIENCY OF CS STRATEGY, REGULAR EAD STRATEGY, AND EEAC STRATEGY

\begin{tabular}{ccccc}
\hline \hline & & Scenario A & & Scenario B \\
& Front axle & Rear axle & Front axle & Rear axle \\
\hline CS strategy & $59.63 \%$ & $55.62 \%$ & $57.98 \%$ & $51.35 \%$ \\
Regular EAD strategy (Upper-stage) & $72.36 \%$ & $70.35 \%$ & $70.33 \%$ & $68.97 \%$ \\
Regular EAD strategy (Lower-stage) & $61.10 \%$ & $58.95 \%$ & $60.55 \%$ & $60.01 \%$ \\
EEAC strategy (Upper-stage) & $74.25 \%$ & $73.21 \%$ & $71.89 \%$ & $70.52 \%$ \\
EEAC strategy (Lower-stage) & $73.69 \%$ & $72.66 \%$ & $70.03 \%$ & $69.91 \%$ \\
\hline \hline
\end{tabular}

In Fig. 10, the prediction error of discharge time is within the range $-13.02 \% \sim 7.41 \%$. Although the maximum error is $13.02 \%$, the mean error is only $3.83 \%$. Besides, Table VII lists the results of several simulations, i.e., the cases result in maximum and minimum vehicle queue discharge time prediction errors. To further investigate the advantages of the proposed IQDP method, the EEAC using the traditional DKM [25] for queue discharge prediction is used as the baseline. The terminal velocity is made identical for all simulated cases so that the comparison can be carried out based only on the battery energy consumption in Table VII.

As observed, the queue discharge prediction accuracy has a significant influence on energy efficiency of the EEAC strategy. The battery energy consumption for cases with maximum queue discharge time prediction error increased by around $20 \%$ compared with that from the long-term global optimization. Besides, the EEAC with DKM shows the worst performance, with the battery energy consumption increased by $33.52 \%$. 
TABLE VI

ENERGY CONSUMPTION OF CS STRATEGY, REGULAR EAD STRATEGY, AND EEAC STRATEGY

\begin{tabular}{|c|c|c|c|c|c|c|}
\hline & \multicolumn{3}{|c|}{ Scenario A } & \multicolumn{3}{|c|}{ Scenario B } \\
\hline & $\begin{array}{l}\text { Battery energy } \\
\text { consumption }\end{array}$ & $\begin{array}{l}\text { Vehicle kinetic } \\
\text { energy changes }\end{array}$ & $\begin{array}{l}\text { Energy efficiency } \\
\text { improvement }\end{array}$ & $\begin{array}{c}\text { Battery energy } \\
\text { consumption }\end{array}$ & $\begin{array}{l}\text { Vehicle kinetic } \\
\text { energy changes }\end{array}$ & $\begin{array}{l}\text { Energy efficiency } \\
\text { improvement }\end{array}$ \\
\hline CS strategy & $249.38 \mathrm{~kJ}$ & $58.20 \mathrm{~kJ}$ & N/A & $194.15 \mathrm{~kJ}$ & $99.48 \mathrm{~kJ}$ & N/A \\
\hline $\begin{array}{l}\text { Regular EAD strategy } \\
\text { (Upper-stage) }\end{array}$ & $175.88 \mathrm{~kJ}$ & $71.71 \mathrm{~kJ}$ & $19.50 \%$ (vs. CS) & $138.67 \mathrm{~kJ}$ & $112.30 \mathrm{~kJ}$ & $14.53 \%$ (vs. CS) \\
\hline $\begin{array}{l}\text { Regular EAD strategy } \\
\text { (Lower-stage) }\end{array}$ & $248.39 \mathrm{~kJ}$ & $57.52 \mathrm{~kJ}$ & $0.54 \%$ (vs. CS) & $203.11 \mathrm{~kJ}$ & $99.48 \mathrm{~kJ}$ & $-3.05 \%$ (vs. CS) \\
\hline $\begin{array}{l}\text { EEAC strategy } \\
\text { (Upper-stage) }\end{array}$ & $182.97 \mathrm{~kJ}$ & $70.56 \mathrm{~kJ}$ & $17.57 \%$ (vs. CS) & $121.42 \mathrm{~kJ}$ & $135.55 \mathrm{~kJ}$ & $12.49 \%$ (vs. CS) \\
\hline $\begin{array}{l}\text { EEAC strategy } \\
\text { (Lower-stage) }\end{array}$ & $197.32 \mathrm{~kJ}$ & $71.86 \mathrm{~kJ}$ & $\begin{array}{c}12.48 \% \text { (vs. CS }) \\
12.01 \% \text { (vs. regular EAD) }\end{array}$ & $134.04 \mathrm{~kJ}$ & $134.61 \mathrm{~kJ}$ & $\begin{array}{c}8.51 \%(\text { vs. CS }) \\
11.22 \%(\text { vs. regular EAD) }\end{array}$ \\
\hline
\end{tabular}

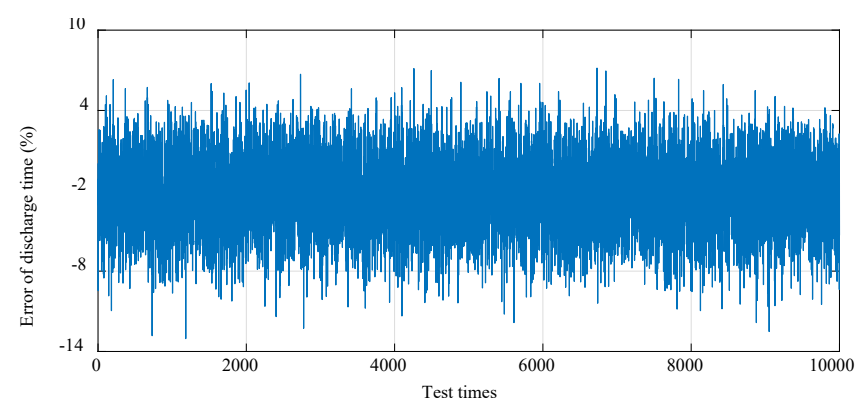

Fig. 10. The prediction error of vehicle queue discharge time.

Table VII

ENERGY CONSUMPTION OF 10,000 SIMULATIONS

\begin{tabular}{cccc}
\hline \hline & \multicolumn{2}{c}{ Battery energy consumption } & Compared with \\
& Upper-stage & Lower-stage & upper-stage result \\
\hline DKM & $179.04 \mathrm{~kJ}$ & $239.05 \mathrm{~kJ}$ & $33.52 \%$ \\
$\begin{array}{c}\text { Minimum discharge } \\
\text { time prediction error }\end{array}$ & $182.97 \mathrm{~kJ}$ & $183.74 \mathrm{~kJ}$ & $0.42 \%$ \\
$\begin{array}{l}\text { Maximum discharge } \\
\text { time prediction error }\end{array}$ & $220.36 \mathrm{~kJ}$ & $20.41 \%$ \\
\hline \hline
\end{tabular}

In summary, the above results demonstrate the vehicle queue discharge prediction accuracy is important to achieve reliable eco-approach control at the signalized intersection. It deserves more investigation in the future.

\section{CONCLUSION}

This paper presents the EEAC strategy to achieve vehicle energy-efficient operation at the signalized intersection with consideration of vehicle queue. First, the vehicle queue discharge movement is predicted by using the proposed IQDP method which considers the vehicle and driver dynamics. Second, a hierarchical control framework is presented, which includes two stages: the upper-stage for long-term global optimization and the lower-stage for short-term adaptation. In the upper-stage, the vehicle energy-efficient speed is calculated by using the temporal-domain DP, which enables efficient and safe vehicle operation while driving through the intersection. In the lower-stage, an online LTV-MPC controller is proposed to follow the optimal speed profile derived from the upper-stage to prevent possible rear-end collision caused by the queue discharge time prediction error.

Simulation results indicated that the EEAC can achieve significant energy efficiency improvement as compared with
$\mathrm{CS}$ and regular EAD since the EEAC can reduce vehicle resistance losses and is able to operate the motor working in a high-efficiency zone by manipulating the driving speed. In addition, the vehicle queue discharge prediction accuracy has a considerable influence on the energy-saving of the EEAC. Thus, it can improve the robustness of EEAC if a more accurate realtime queue discharge prediction method is employed.

There are some directions for future work. One is to relax the study of dynamic traffic influences, such as actuated traffic signal control logic and nonstationary traffic flow at the intersection. It is also promising to integrate data-driven prediction approaches with real-world traffic data.

\section{REFERENCES}

[1] S. Davis, and R. Boundy, Transportation Energy Data Book: Edition 38, U.S. Dept. Energy, Washington, DC, USA, 2020. doi:10.2172/1606919.

[2] W. Zhang, S. E. Li, X. Zhang, D. Kum, Z. Song, G. Yin, and $\mathrm{F}$. Ju, "A survey of powertrain configuration studies on hybrid electric vehicles," Applied Energy, vol. 262, pp. 117, Mar. 2020.

[3] J. J. Eckert, L. C. d. A. Silva, F. G. Dedini, and F. C. Corrêa, "Electric vehicle powertrain and fuzzy control multiobjective optimization, considering dual hybrid energy storage systems," IEEE Transactions on Vehicular Technology, vol. 69, no. 4, pp. 3773-3782, Apr. 2020.

[4] A. Desreveaux, A. Bouscayrol, R. Trigui, E. Castex, and J. Klein, "Impact of the velocity profile on energy consumption of electric vehicles," IEEE Transactions on Vehicular Technology, vol. 68, no. 12, pp. 11420-11426, Dec. 2019.

[5] N. Jollands, P. Waide, M. Ellis, T. Onoda, J. Laustsen, K. Tanaka, P. de TSerclaes, I. Barnsley, R. Bradley, and A. Meier, "The 25 IEA energy efficiency policy recommendations to the G8 Gleneagles Plan of Action," Energy policy, vol. 38, no. 11, pp. 6409-6418, Nov. 2010.

[6] A. Vahidi and A. Sciarretta, "Energy-saving potentials of connected and automated vehicles," Transportation Research Part C. Emerging Technologies, vol. 95, pp. 822843, Oct. 2018.

[7] J. Kim and C. Ahn, "Real-time speed trajectory planning for minimum fuel consumption of a ground vehicle," IEEE Transactions on Intelligent Transportation Systems, vol. 21, no. 6, pp. 2324-2338, Jun. 2020. 
[8] S. E. Li, R. Li, J. Wang, X. Hu, B. Cheng, and K. Li, "Stabilizing periodic control of automated vehicle platoon with minimized fuel consumption," IEEE Transactions on Transportation Electrification, vol. 3, no. 1, pp. 259-271, Mar. 2017.

[9] W. Zhuang, L. Qu, S. Xu, B. Li, C. Chen, and G. Yin, "Integrated energy-oriented cruising control of electric vehicle on highway with varying slopes considering battery aging," Science China Technological Sciences, vol.63, no.1, pp.155-165, Nov. 2019.

[10] F. Ding and H. Jin, "On the optimal speed profile for ecodriving on curved roads," IEEE Transactions on Intelligent Transportation Systems, vol. 19, no. 12, pp. 4000-4010, Dec. 2018.

[11] S. Zhang, Y. Luo, J. Wang, X. Wang, and K. Li, "Predictive energy management strategy for fully electric vehicles based on preceding vehicle movement," IEEE Transactions on Intelligent Transportation Systems, vol. 18, no. 11, pp. 3049-3060, Nov. 2017.

[12] S. Xu and H. Peng, "Design and comparison of fuel-saving speed planning algorithms for automated vehicles," IEEE Access, vol. 6, pp. 9070-9080, Feb. 2018.

[13] Q. Guo, L. Li, and X. J. Ban, "Urban traffic signal control with connected and automated vehicles: a survey," Transportation research part C: emerging technologies, vol. 101, pp. 313-334, Apr. 2019.

[14] K. Katsaros, R. Kernchen, M. Dianati, and C. Zinoviou, "Application of vehicular communications for improving the efficiency of traffic in urban areas," Wireless Communications and Mobile Computing, Vol. 11, 2011,pp. 1657-1667, Dec. 2011.

[15] G. Mahler and A. Vahidi, "An optimal velocity-planning scheme for vehicle energy efficiency through probabilistic prediction of traffic-signal timing," IEEE Transactions on Intelligent Transportation Systems, vol. 15, no. 6, pp. 2516-2523, Dec. 2014.

[16] Q. Lin, S. Li, X. Du, X. Zhang, H. Peng, Y. Luo, and K. $\mathrm{Li}$, "Minimize the fuel consumption of connected vehicles between two red-signalized intersections in urban traffic," IEEE Transactions on Vehicular Technology, vol. 67, no. 10, pp. 9060-9072, Oct. 2018.

[17] Z. Wang, Y. Bian, S. E. Shladover, G. Wu, S. E. Li, and M. J. Barth, "A survey on cooperative longitudinal motion control of multiple connected and automated vehicles," IEEE Intelligent Transportation Systems Magazine, vol. 12, no. 1, pp. 4-24, Dec. 2020.

[18] S. E. Li, S. Xu, X. Huang, B. Cheng, and H. Peng, "Ecodeparture of connected vehicles with V2X communication at signalized intersections," IEEE Transactions on Vehicular Technology, vol. 64, no. 12, pp. 5439-5449, Dec. 2015.

[19] L. Xie, Y. Luo, D. Zhang, R. Chen, and K. Li, "Intelligent energy-saving control strategy for electric vehicle based on preceding vehicle movement," Mechanical Systems and Signal Processing, vol. 130, pp. 484-501, Sept. 2019.

[20] F. Ye, P. Hao, X. Qi, G. Wu, K. Boriboonsomsin, and M. J. Barth, "Prediction-based eco-approach and departure at signalized intersections with speed forecasting on preceding vehicles," IEEE Transactions on Intelligent
Transportation Systems, vol. 20, no. 4, pp. 1378-1389, Apr. 2019

[21] A. Bakibillah, M. Kamal, C. Tan, T. Hayakawa, and J. Imura, "Event-driven stochastic eco-driving strategy at signalized intersections from self-driving data," IEEE Transactions on Vehicular Technology, vol. 68, no. 9, pp. 8557-8569, Sept. 2019.

[22] X. He, X. Liu, and H. Liu, "Optimal vehicle speed trajectory on a signalized arterial with consideration of queue," Transportation Research Part C: Emerging Technologies, vol. 61, pp. 106-120, Dec. 2015.

[23] A. Adriano, A.Campagna, P. Site, F. Filippi, and L. Persia, "Automated vehicles and the rethinking of mobility and cities," Transportation Research Procedia, vol. 5, pp. 145160, Feb. 2015.

[24] H. Yang, F. Almutairi, and H. Rakha, "Eco-driving at signalized intersections: a multiple signal optimization approach," IEEE Transactions on Intelligent Transportation Systems, pp. 1-13, Mar. 2020. doi: 10.1109/TITS.2020.2978184.

[25] H. Liu, X. Y. Lu, and S. Shladover, "Traffic signal control by leveraging Cooperative Adaptive Cruise Control (CACC) vehicle platooning capabilities," Transportation Research Part C: Emerging Technologies, vol. 104, pp. 390-407, Jul. 2019.

[26] S. Yu, R. Fu, Y. Guo, Q. Xin, and Z. Shi, "Consensus and optimal speed advisory model for mixed traffic at an isolated signalized intersection," Physica A: Statistical Mechanics and its Applications, vol. 531, no. 1, pp. 1-22, Oct. 2019.

[27] H. Yang, H. Rakha, and M. Ala, "Eco-cooperative adaptive cruise control at signalized intersections considering queue effects," IEEE Transactions on Intelligent Transportation Systems, vol. 18, no. 6, pp. 1575-1585, Jun. 2017.

[28] Z. Yang, Y. Feng, X. Gong, D. Zhao, and J. Sun, "Ecotrajectory planning with consideration of queue along congested corridor for hybrid electric vehicles," Transportation Research Record, vol. 2673, no. 9, pp. 277286, May 2019.

[29] M. Zhou, Y. Yu, and X. Qu, "Development of an efficient driving strategy for connected and automated vehicles at signalized intersections: a reinforcement learning approach," IEEE Transactions on Intelligent Transportation Systems, vol. 21, no. 1, pp. 433-443, Jan. 2020.

[30] C. Sun, J. Guanetti, F. Borrelli, and S. J. Moura, "Optimal eco-driving control of connected and autonomous vehicles through signalized intersections," IEEE Internet of Things Journal, vol. 7, no. 5, pp. 3759-3773, May 2020.

[31] J. Zhang, W. Sun, and H. Du, "Integrated motion control scheme for four-wheel-independent vehicles considering critical conditions," IEEE Transactions on Vehicular Technology, vol. 68, no. 8, pp. 7488-7497, Aug. 2019.

[32] H. Peng, W. Wang, C. Xiang, L. Li, and X. Wang, "Torque coordinated control of four in-wheel motor independentdrive vehicles with consideration of the safety and economy," IEEE Transactions on Vehicular Technology, vol. 68, no. 10, pp. 9604-9618, Oct. 2019. 
[33] F. Ju, W. Zhuang, L. Wang, and Z. Zhang, "Comparison of four-wheel-drive hybrid powertrain configurations," Energy, vol. 209, pp. 1-14, Oct. 2020.

[34] F. Biral, M. Da Lio, and E. Bertolazzi, "Combining safety margins and user preferences into a driving criterion for optimal control-based computation of reference maneuvers for an ADAS of the next generation," IEEE Intelligent Vehicles Symposium, Las Vegas, NV, USA, 2005, pp. 3641.

[35] Z. Wang, Y. Wang, Z. Gao, and J. Gao, "Torque distribution control strategy based on dynamic axle load for 8 in-wheel motor drive vehicle," Energy Procedia, vol. 104, pp. 550-555, Dec. 2016.

[36] C. Kumar and S. Subramanian, "Cooperative control of regenerative braking and friction braking for a hybrid electric vehicle," Proceedings of the Institution of Mechanical Engineers Part D: Journal of Automobile Engineering, vol. 230, no. 1, pp. 103-116, Apr. 2016.

[37] N. Polson and V. Sokolov, "Deep learning for short-term traffic flow prediction," Transportation Research Part C: Emerging Technologies, vol. 79, pp. 1-17, Jun. 2017.

[38] R. E. Bellman and S. E. Dreyfus, Applied Dynamic Programming. Princeton, NJ, USA: Princeton Univ. Press, 2015.

[39] B. Asadi and A. Vahidi, "Predictive cruise control: utilizing upcoming traffic signal information for improving fuel economy and reducing trip time," IEEE Transactions on Control Systems Technology, vol. 19, no. 3, pp. 707-714, May 2011.

[40] P. Falcone, F. Borrelli, H. E. Tseng, J. Asgari, and D. Hrovat, "Linear time-varying model predictive control and its application to active steering systems: stability analysis and experimental validation," International Journal of Robust and Nonlinear Control, vol.18, no.8, pp. 862-875, Jul. 2007.

[41] Z. Chen, C.C. Mi, R. Xiong, J. Xu, C. You. "Energy management of a power-split plug-in hybrid electric vehicle based on genetic algorithm and quadratic programming,". Journal of Power Sources, 2014, vol. 248, no. 7, pp. 416-426, Feb. 2014.

[42] M. Treiber, A. Hennecke, and D. Helbing, "Congested traffic states in empirical observations and microscopic simulations," Physical review E, vol. 62, no. 2, pp. 18051824, Aug. 2000.

[43] A. Kesting, M. Treiber, M. Schönhof, and D. Helbing, "Extending adaptive cruise control to adaptive driving strategies," Transportation Research Record, vol. 2000, no. 1, pp. 16-24, Jan. 2007.

[44] S. Shladover, D. Su, and X. Lu. "Impacts of cooperative adaptive cruise control on freeway traffic flow," Transportation Research Record, vol. 2324. no. 1, pp. 6370, Jan. 2012.

[45] S. E. Li and H. Peng, "Strategies to minimize the fuel consumption of passenger cars during car-following scenarios," Proceedings of the Institution of Mechanical Engineers, Part D: Journal of Automobile Engineering, vol. 226, no. 3, pp. 419-429, Mar. 2012.

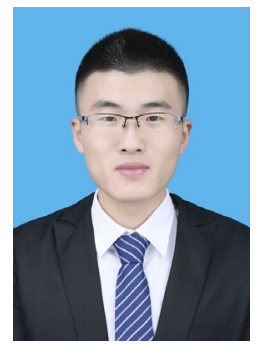

Haoxuan Dong received the M.S. degree in vehicle engineering from Chang'an University, Xi'an, China, in 2018. He is currently pursuing the Ph.D. degree with the Southeast University, Nanjing, China.

His current research interests include connected and automated vehicles, energyefficient driving, vehicle dynamics control.

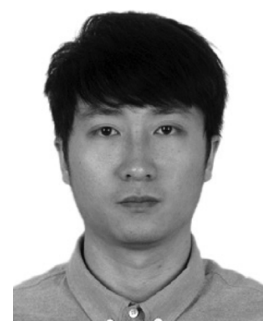

Weichao Zhuang received the B.S. and $\mathrm{Ph} . \mathrm{D}$. degrees in mechanical engineering from the Nanjing University of Science and Technology, Nanjing, China, in 2012 and 2017, respectively. From 2014 to 2015, he was a Visiting Student with the Department of Mechanical Engineering, University of Michigan, Ann Arbor, MI, USA. He is currently an Assistant Professor with the School of Mechanical Engineering, Southeast University, Nanjing.

His current research interests include, optimal control, clean energy vehicles, connected vehicles, and multiagent control.

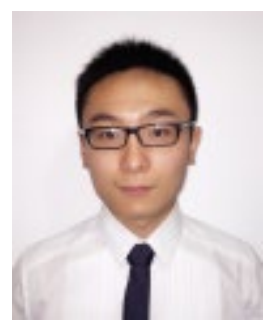

Boli Chen (M'16) received the B. Eng. in Electrical and Electronic Engineering in 2010 from Northumbria University, UK. In 2011 and 2015, he respectively received the MSc and the Ph.D. in Control Systems from Imperial College London, UK. Currently, he is a Lecturer in the Department of Electronic and Electrical Engineering, University College London, U.K.

His research focuses on control, optimization, estimation and identification of a range of complex dynamical systems, mainly from automotive and power electronics areas.

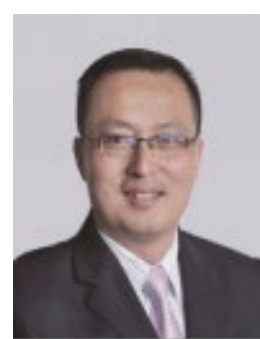

Guodong Yin (M'15-SM'20) received the Ph.D. degree in vehicle engineering from Southeast University, Nanjing, China, in 2007. From 2011 to 2012, he was a Visiting Research Scholar with the Department of Mechanical and Aerospace Engineering, Ohio State University, Columbus, OH, USA. He is currently a Professor with the School of Mechanical Engineering, Southeast University.

His current research interests include vehicle dynamics and control, connected vehicles, and multiagent control.

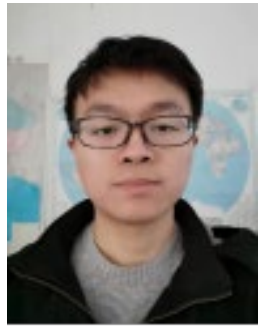

Yan Wang received the M.S. degrees in vehicle engineering from the Nanjing University of Aeronautics and Astronautics, Nanjing, China, in 2018. He is currently pursuing a Ph.D. degree with the Southeast University, Nanjing, China.

His current research interests include vehicle state and parameter estimation, automotive active safety control. 